\title{
BEAM TRANSFER FUNCTIONS AND BEAM STABILISATION IN A DOUBLE RF SYSTEM
}

\author{
E. Shaposhnikova, T. Bohl and T. Linnecar, CERN, Geneva, Switzerland
}

\begin{abstract}
The high intensity proton beam for LHC accelerated in the CERN SPS is stabilised against coupled-bunch instabilities by a 4th harmonic RF system in bunch-shortening mode. Bunch-lengthening mode, which could also be useful to reduce peak line density and alleviate problems from e-cloud and kicker heating, does not give desirable results for beam stability. In this paper an analysis of the limitations of these two different modes of operation is presented together with measurements of the Beam Transfer Function for the double RF system. As predicted by theory, for sufficiently long bunches with the same noise excitation, the measured amplitude of the beam response in bunchlengthening mode is an order of magnitude higher than that for bunch-shortening mode or for a single RF system.
\end{abstract}

\section{BEAM STABILISATION}

Double RF systems are widely used for high intensity beams to modify the particle or synchrotron frequency distribution inside the bunch. In low energy proton rings the higher harmonic RF system is usually added to flatten bunches to reduce the space charge effects. This mode of operation gives an increase in synchrotron frequency spread $\Delta \omega_{s}$ by decreasing the zero-amplitude frequency $\omega_{s}(0)$ (for maximum effect to zero) [1], and is often called bunch-lengthening (BL) mode. An increase in $\Delta \omega_{s}$, to cure coupled-bunch instabilities, can also be achieved in bunchshortening (BS) mode, by increasing $\omega_{s}(0)$. The total voltage in the double RF system is

$$
V=V_{1} \sin (\phi)+V_{2} \sin \left(h_{2} \phi / h_{1}+\Phi_{2}\right),
$$

where $V_{1,2}$ and $h_{1,2}$ are the voltage amplitudes and harmonic numbers of the main and high frequency RF systems and $\phi$ is the phase deviation from the synchronous phase $\phi_{s 0}$ for $V_{2}=0$. For a non-accelerating bucket the maximum increase in $\Delta \omega_{s}$ is obtained when $\Phi_{2}=0, \pi$ (correspondingly $\mathrm{BL}$ and $\mathrm{BS}$ mode above transition, $\phi_{s o}=\pi$ ). Below we consider only these two cases with $h_{2} / h_{1}=4$.

In recent years a high intensity beam was accelerated in the CERN SPS in preparation for both the LHC and CNGS projects. An impedance reduction programme allowed the microwave instability to be eliminated up to nominal LHC intensities of $1.15 \times 10^{11} / \mathrm{bunch}$. Nevertheless the high intensity beam still suffers from strong coupled-bunch instabilities during the ramp at high energies. Despite the fact that for the LHC beam the instability threshold is five times below the nominal bunch intensity, in 2003 the nominal longitudinal parameters were obtained at top energy,
$450 \mathrm{GeV}$ [2]. Beam stabilisation was achieved by operating the fourth harmonic RF system in BS-mode throughout the cycle in addition to the main $200 \mathrm{MHz}$ RF system and by controlled emittance blow-up ( $\sim 25 \%)$ at high energy.

The experimental fact that we were unable to stabilise either the fixed-target beam in the past [3], or more recently the LHC beam [2], using BL-mode on the ramp, could be explained by the very tight requirement on the relative phase of the two RF systems which are difficult to satisfy in the presence of beam loading [3]. Problems with beam stability in BL-mode have also been seen in the CERN Booster [4]. This is similar to experience in HERA [5], where BS-mode is used in operation. Beam stability in a double RF system has also been studied in [6].

Nevertheless BL-mode is still interesting in the SPS, due to the resulting decrease in particle density which reduces e-cloud effects and the heating of ferrite elements, critical for nominal LHC and CNGS beams [7]. At the end of the 2004 beam run we used the $26 \mathrm{GeV} / \mathrm{c}$ flat bottom in the SPS, where accurate adjustment of the phase shift $\Phi_{2}$ between the two RF systems could be made, to study the effect of BL-mode on the $75 \mathrm{~ns}$ spaced LHC beam which suffers less from beam loading than the nominal $25 \mathrm{~ns}$ spaced beam. With $V_{1}=3 \mathrm{MV}$ and $V_{2}=0.7 \mathrm{MV}$ an accuracy of $\pm 0.2 \mathrm{rad}$ in $\Phi_{2}$ is required to obtain the same $\Delta \omega_{s}$ in $\mathrm{BL}$ and BS modes. Measurements of the beam transfer functions (BTF) were also used to achieve accurate setting of phase $\Phi_{2}$. However, we were again unable to stabilise this beam in BL-mode. A weak instability seen with $V_{2}=0$ (Fig. 1, top) became even more pronounced in BL-mode (middle). Beam of the same intensity was stable in BS mode (bottom). The $200 \mathrm{MHz}$ voltage $V_{1}$ was ramped up from 1.85 MV at injection to $3 \mathrm{MV}$ along the flat bottom and $V_{2}$ was a constant $0.7 \mathrm{MV}$. The error bar magnitude in Fig. 1 is defined by differences in bunch intensity.

Measurements of BTF, presented in the following section, point to a local loss of Landau damping, typical for BL-mode, as a probable explanation for this reduction of beam stability threshold.

\section{BEAM TRANSFER FUNCTIONS}

The beam transfer functions were measured using a low frequency network analyser. The beam was excited by adding noise to the $200 \mathrm{MHz}$ cavity voltage reference. The quadrupole excitation of the beam was measured by peak amplitude detection of the longitudinal pick-up signal of the first bunch. The measurement time was $800 \mathrm{~ms}$ for a frequency span of $1 \mathrm{kHz}$. The measurements were taken $5 \mathrm{~s}$ after injection and the data of $(\sim 10)$ consecutive cycles 

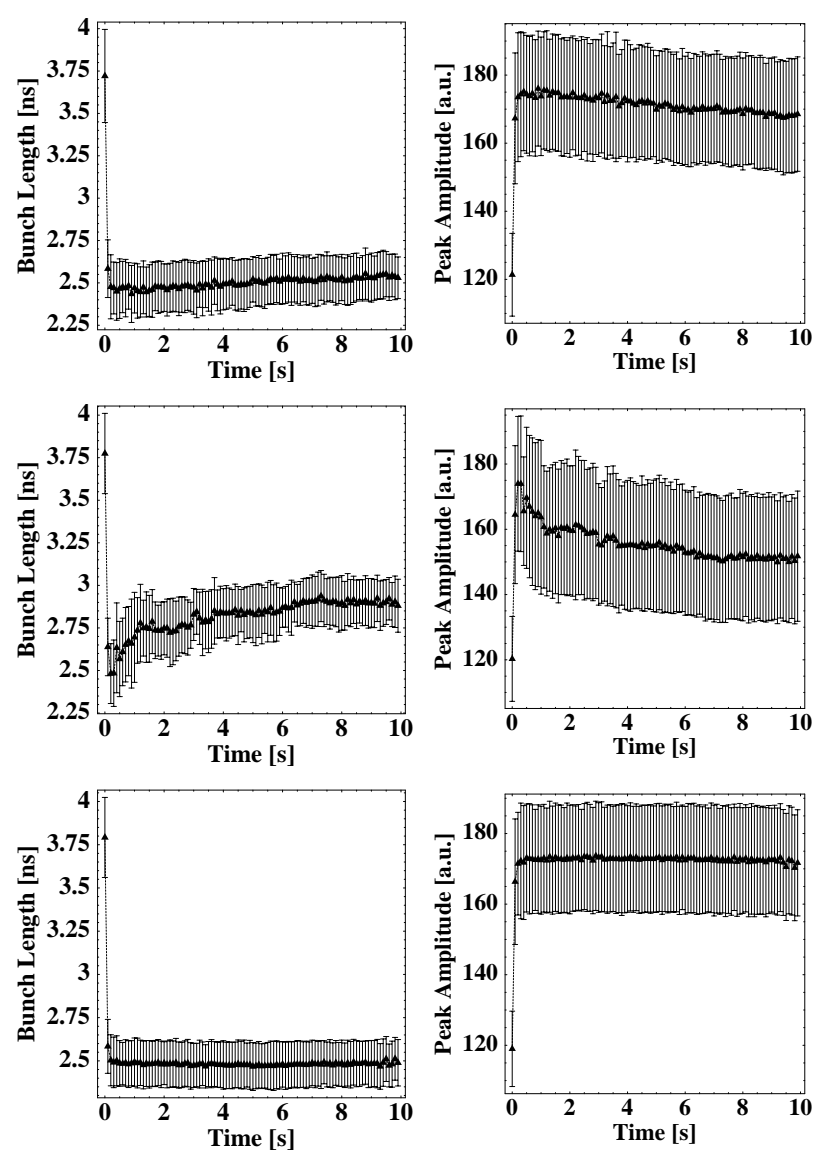

Figure 1: The average ( 24 bunches) bunch length (left) and peak amplitude (right) on the SPS flat bottom at $26 \mathrm{GeV} / \mathrm{c}$ with the $800 \mathrm{MHz}$ RF system off (top) and $800 \mathrm{MHz}$ in BL (middle) and in BS (bottom) mode. Average bunch intensity $\bar{N}_{b}=1.25 \times 10^{11}, V_{1}=3 \mathrm{MV}, V_{2}=0.7 \mathrm{MV}$.

were averaged to obtain a reasonable signal/noise ratio.

The BTF amplitude and phase measured at the same time and with the same experimental conditions as in Fig. 1, are shown in Fig. 2 (raw data). As can be seen the signal amplitude for the case of BL mode is an order of magnitude higher than for a single RF system or BS mode.

This large amplitude signal is related to the existence of a region where the derivative of the synchrotron frequency as a function of action $J$ equals zero, $\omega_{s}^{\prime}(J)=0$. Landau damping in this region is lost and a perturbation grows $\propto \sqrt{t}$. Detailed calculations of BTF in single and double RF systems are presented in [8]. Below we consider only the case related to our measurements with a potential well symmetric in $\phi$. No intensity effects are taken into account.

The amplitude of the main harmonic of the beam current perturbation $\Delta I_{1}(\omega)$ is connected to the main voltage amplitude modulation $\Delta V_{1}(\omega)$ by

$$
\Delta I_{1}(\omega)=I_{0} \sum_{m=2,4 \ldots}^{\infty} \frac{m^{2}}{k} M_{m}^{11}(\omega) \Delta V_{1}(\omega) / V_{1},
$$

where $I_{0}$ is the average beam current. The matrix element $M_{m}^{p k}(\omega)$ is proportional to the dispersion integral and
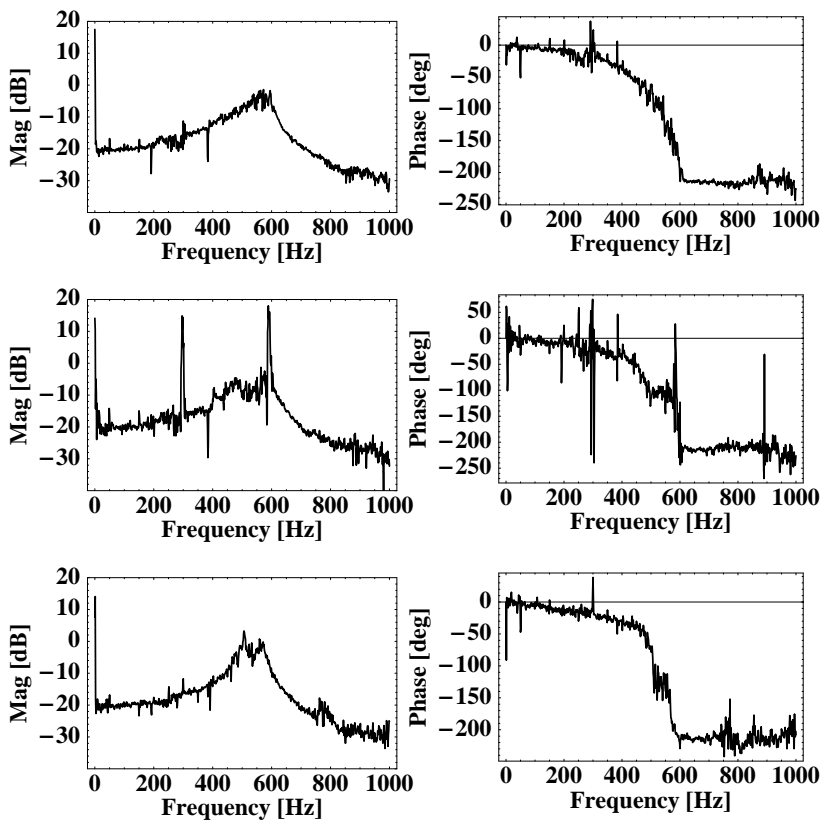

Figure 2: BTF amplitude (left) and phase (right) measured on the $26 \mathrm{GeV} / \mathrm{c}$ flat bottom in the SPS for single (top) and double RF system in BL (middle) and BS (bottom) operation modes (as in Fig.1), $\omega_{s 0} /(2 \pi)=313 \mathrm{~Hz}$.

contains information about the amplitude and phase of the beam response with respect to the excitation

$$
M_{m}^{p k}(\omega) \propto \int_{0}^{J_{\max }} \frac{d \mathcal{F}_{0}}{d J} \frac{I_{m p}(J) I_{m k}(J) \omega_{s}(J) d J}{(\omega-i \sigma)^{2}-m^{2} \omega_{s}^{2}(J)},
$$

where the integration contour is chosen to satisfy the initial conditions, $\mathcal{F}_{0}(J)$ is the initial particle distribution function and $I_{m k}(J)$ are the coefficients from the Fourier expansion of a plane wave over the phase of the synchrotron oscillations $\psi[9]$

$$
I_{m k}(J)=\frac{1}{2 \pi} \int_{-\pi}^{\pi} \exp \left[\frac{k}{h_{1}} \phi(\psi, J)+m \psi\right] d \psi .
$$

For positive $\omega$ and dependence $\omega_{s}(J)$ inside the bunch such that $\omega_{s}^{\prime}\left(J_{0}\right)=0$ only if $d \mathcal{F}_{0} / d J=0$,

$$
\begin{aligned}
M_{m}^{p k}(\omega) & \propto \mathcal{P} \int_{0}^{J_{\max }} \frac{d \mathcal{F}_{0}}{d J} \frac{I_{m k}(J) I_{m p}(J) \omega_{s}(J) d J}{\omega^{2}-m^{2} \omega_{s}^{2}(J)}+ \\
& +\left.i \frac{\pi}{2 m^{2}} \frac{d \mathcal{F}_{0}}{d J}\right|_{J=J_{0}} \frac{I_{m k}\left(J_{0}\right) I_{m p}\left(J_{0}\right)}{\left|\omega_{s}^{\prime}\left(J_{0}\right)\right|}
\end{aligned}
$$

where $\mathcal{P}$ means the principal value of the integral.

For long enough bunches in a double RF system the case where $\omega_{s}^{\prime}\left(J_{c r}\right)=0$ for some $J=J_{c r}$ exists in BL-mode and also in BS-mode for large enough $V_{2}$. For an excitation frequency $\omega$ which satisfies the resonant condition $\delta \omega=$ $\omega-m \omega_{s}\left(J_{c r}\right)=0$ the integral (3) diverges as $1 / \sqrt{\delta \omega}$ [8] and, strictly speaking, the linear perturbation theory used to calculate the BTF is no longer applicable. Since the same dispersion integrals appear in beam stability analysis it has 


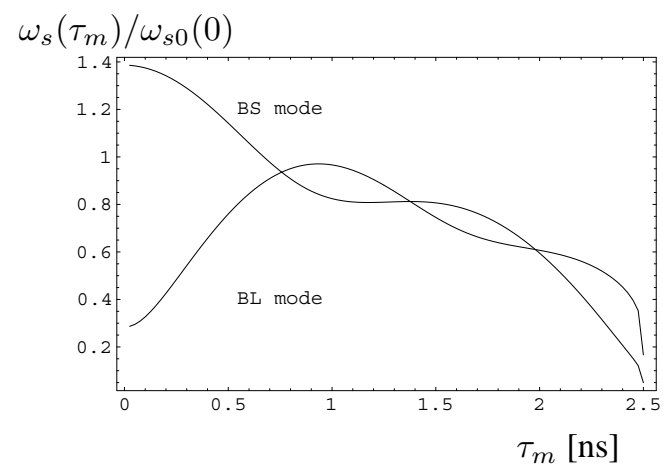

Figure 3: Normalised synchrotron frequency as a function of synchrotron oscillation amplitude for $V_{2} / V_{1}=0.23$.
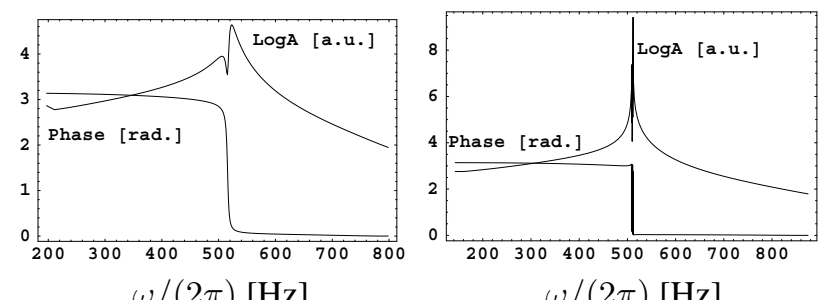

$\omega /(2 \pi)[\mathrm{Hz}]$

$\omega /(2 \pi)[\mathrm{Hz}]$

Figure 4: Calculated amplitude and phase of $M_{2}^{11}(\omega)$ in BS-mode for Gaussian distribution with $\sigma_{t}=0.6 \mathrm{~ns}$ and $V_{2} / V_{1}=0.15$ (left) and 0.23 (right).

been suggested [10] that the threshold intensity in this case is zero.

The synchrotron frequency as a function of the synchrotron oscillation amplitude $\tau_{m}$ (in ns) for BS and BL modes is shown in Fig. 3. For a $4 \sigma$ bunch length of $2.5 \mathrm{~ns}$ in our experiment (Fig. 1) $\tau_{m}=1.25 \mathrm{~ns}$ and in BLmode the region with $\omega_{s}^{\prime}\left(J_{c r}\right)=0$ is inside the bunch. In BS-mode this region appears only for $V_{2} / V_{1} \geq 0.2$ and for $4 \sigma=2.5 \mathrm{~ns}$ is just inside the bunch tails, where $d \mathcal{F}_{0} / d J \sim 0$. The BTF $M_{2}^{11}(\omega)$ calculated using equations (3-5) for $V_{2} / V_{1}=0.15,0.23$ is presented in Fig. 4. Comparison with Fig. 2 suggests that in our experiment $V_{2} / V_{1}$ was $\sim 10 \%$ lower than estimated (less than 0.2 ) or the bunch was slightly shorter.

No instability specific to BL-mode was observed at low intensities even with the large amplitude signal in BTFs measured with a fixed target (CNGS) beam during the coast at $120 \mathrm{GeV} / \mathrm{c}$ in 1998 (Fig. 5). However the change to the equilibrium bunch distribution (creation of shoulders in regions where $d \mathcal{F}_{0} / d J=0$ ) from the beginning to the end of the coast can be clearly seen in Fig. 6. A similar effect was observed in numerical simulations for a double RF system with space-charge effects included [11].

Summary. Degradation of beam stability in BL-mode has been observed for high intensity beams both during acceleration [2], [3] and now on the flat bottom even with a spread $\Delta \omega_{s}$ five times larger than in a single RF system. Measurements of BTF show a very strong coherent beam response in BL-mode at frequencies corresponding

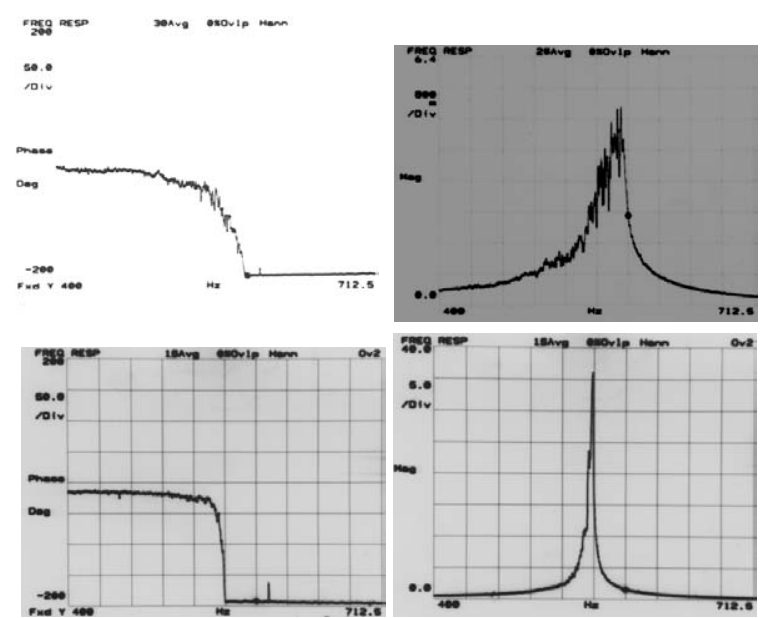

Figure 5: BTF phase (left) and amplitude (right) measured at $120 \mathrm{GeV} / \mathrm{c}$ in single (top, vertical scale $\mathbf{0 . 5} \mathbf{~ V}$ ) and double RF system in BL-mode (bottom, vertical scale $\mathbf{5} \mathbf{~ V}$ ). $\bar{N}_{b}=$ $0.5 \times 10^{10}, V_{1}=3.3 \mathrm{MV}$ and $V_{2}=0.3 \mathrm{MV}$.
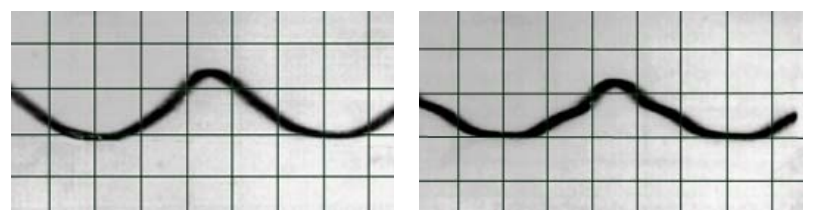

Figure 6: Bunch profiles at the beginning (left) and the end (right) of the 10 min coast at $120 \mathrm{GeV} / \mathrm{c}$ in $\mathrm{BL}$ mode from Fig. 5. Horizontal scale $1 \mathrm{~ns} /$ div.

to $\omega_{s}^{\prime}(J)=0$. However final confirmation that such a region can be responsible for the decrease in the instability threshold would be by obtaining a similar result in BSmode (with longer bunches and larger $V_{2} / V_{1}$ ratios). This is planned for future studies.

We are grateful to J. Tuckmantel, J. F. Malo and U. Wehrle for their help.

\section{REFERENCES}

[1] A. Hofmann and S. Myers, Proc. 11th Int. Conf. HighEnergy Acc., Geneva, 1980, p. 610.

[2] P. Baudrenghien et al., Proc. PAC 2003, p. 3050.

[3] T. Bohl et al., Proc. EPAC 1998, p. 978.

[4] A. Blas, S. Koscielniak, F. Pedersen, CERN Report PS 98028/RF, 1998.

[5] F. Willeke, private communication.

[6] N. Towne, Phys. Rev. ST Accel. Beams 4, 114401 (2001).

[7] G. Arduini et al., Proc. EPAC 2004, p. 623.

[8] E. Shaposhnikova, CERN Report SL/94-19 (RF), 1994.

[9] A. N. Lebedev, Atom. Energ. 25, p. 100, 1968.

[10] V. I. Balbekov, S. V. Ivanov, Atom. Energ. 62 (2), p. 98, 1987.

[11] O. Boine-Frankenheim, T. Shukla, Phys. Rev. ST Accel. Beams 8, 034201 (2005). 